\title{
Bounds on Ratios of Means
}

\section{G. T. Cargo ${ }^{1}$ and O. Shisha}

(August 14, 1962)

\begin{abstract}
The purpose of this paper is to obtain upper bounds for ratios of weighted means $M_{r},-\infty<r<\infty$ (see Hardy, Littlewood, and Polya's "Inequalities," Chapter II). The inequalities arrived at are generalizations of that of Kantorovich.
\end{abstract}

1. Let $q_{1}, q_{2}, \ldots, q_{n}(n>1)$ be positive numbers with $\sum_{k=1}^{n} q_{k}=1$. For every sequence $\left(x_{1}, x_{2}, \ldots, x_{n}\right)$ with all $x_{k}>0$ and for every real $r$, we consider the mean of order $r, M_{r}\left(x_{1}, x_{2}, \ldots, x_{n}\right)$, defined as $\left(\sum_{k=1}^{n} q_{k} x_{k}^{r}\right)^{1 / r}$ if $r \neq 0$, and as $\prod_{k=1}^{n} x_{k}^{q}$ if $r=0$. For given positive $x_{1}, x_{2}, \ldots, x_{n}$ it is known (see, e.g., [1, p. $17]^{2}$ or $\left[2\right.$, p. 26]) that $M_{r}\left(x_{1}, x_{2}, \ldots, x_{n}\right)$ is strictly increasing with $r$ (except when $x_{1}=x_{2}=\ldots=x_{n}$ ).

2 . Let $r, s, A, B$ be given real numbers $(0<A<B$, $r<s)$, and consider the ratios $M_{s}\left(x_{1}, x_{2}, \ldots, x_{n}\right)$ / $M_{r}\left(x_{1}, x_{2}, \ldots, x_{n}\right)$ where each $x_{k}$ varies in the closed interval $[A, B]$. By the above, these ratics are all at least 1 . Our aim is to determine an upper bound for them. In the special case $r=-1, s=1$, which is of importance for applications, this upper bound is given by the Kantorovich inequality $\left(q_{1} x_{1}+\ldots++\right.$ $\left.q_{n} x_{n}\right)\left(q_{1} x_{1}^{-1}+\ldots+q_{n} x_{n}^{-1}\right) \leq(A+B)^{2} /(4 A B)$. For a discussion of this inequality, see [3], where further references are given.

3. Theorem. Let $\mathrm{r}, \mathrm{s}, \mathrm{A}, \mathrm{B}$ be given real numbers $(0<\mathrm{A}<\mathrm{B}, \mathrm{r}<\mathrm{s})$, set $\gamma=\mathrm{B} / \mathrm{A}$, and let $\mathrm{I}$ denote the $\mathrm{n}$-dimensional cube $\left\{\left(\mathrm{x}_{1}, \mathrm{x}_{2}, \ldots, \mathrm{x}_{\mathrm{n}}\right): \mathrm{A} \leq \mathrm{x}_{\mathrm{k}} \leq \mathrm{B}\right.$; $\mathrm{k}=1,2, \ldots, \mathrm{n}\}$. Then

$$
M_{s}\left(x_{1}, x_{2}, \ldots, x_{n}\right) / M_{r}\left(x_{1}, x_{2}, \ldots, x_{n}\right) \leq \Delta
$$

throughout $\mathrm{I}$,

where $\Delta$ is

$$
\left\{\frac{r\left(\gamma^{s}-\gamma^{\gamma}\right)}{(s-r)\left(\gamma^{r}-1\right)}\right\}^{1 / s}\left\{\frac{s\left(\gamma^{r}-\gamma^{s}\right)}{(r-s)\left(\gamma^{s}-1\right)}\right\}^{-1 / r}
$$

$$
\left\{\frac{\gamma^{s /\left(\gamma^{s}-1\right)}}{e \log \left\{\gamma^{s /\left(\gamma^{s}-1\right)}\right\}}\right\}^{1 / s} \quad \text { if } \mathrm{r}=0
$$

and

Let $\theta$ be.

$$
\left\{\frac{\gamma^{r /\left(\gamma^{r}-1\right)}}{e \log \left\{\gamma^{r /\left(\gamma^{r}-1\right)}\right\}}\right\}^{-1 / r} \quad \text { if } \mathrm{s}=0 .
$$

$$
\left\{r /\left(\gamma^{r}-1\right)-s /\left(\gamma^{s}-1\right)\right\} /(s-r) \quad \text { if } \mathrm{rs} \neq 0,
$$

1 This author's contribution was made under a National Academy of SciencesNational Research Council Postdoctoral Resident Research Associateship at the National Bureau of Standards, 1961-62, while on leave from Syracuse University.

${ }_{2}$ Figures in brackets indicate the literature references at the end of this paper.

$$
\frac{1}{s \log \gamma}-\frac{1}{\gamma^{s}-1} \quad \text { if } \mathrm{r}=9
$$

and

$$
\frac{1}{r \log \gamma}-\frac{1}{\gamma^{\prime}-1} \quad \text { if } \mathrm{s}=0 .
$$

Then $0<\theta<1$. Equality in (1) for a point $\left(\mathrm{x}_{1}, \mathrm{x}_{2}\right.$, $\left.\ldots, \mathrm{x}_{\mathrm{n}}\right) \epsilon \mathrm{I}$ holds if and only if there exists a subsequence $\left(\mathrm{k}_{1}, \mathrm{k}_{2}, \ldots, \mathrm{k}_{\mathrm{p}}\right)$ of $(1,2, \ldots, \mathrm{n})$ such that $\sum_{\mathrm{m}=1}^{\mathrm{p}} \mathrm{q}_{\mathrm{k}_{\mathrm{m}}}$ $=\theta, \mathrm{x}_{\mathrm{k}_{\mathrm{m}}}=\mathrm{B}(\mathrm{m}=1,2, \ldots, \mathrm{p})$, and $\mathrm{x}_{\mathrm{k}}=\mathrm{A}$, for every $\mathrm{k}$ distinct from all $\mathrm{k}_{\mathrm{m}}$.

Proof. For every $\left(x_{1}, x_{2}, \ldots, x_{n}\right) \in I$, let $\begin{aligned} F\left(x_{1}, x_{2}, \ldots, x_{n}\right) & \stackrel{.}{=} M_{s}\left(x_{1}, x_{2}, \ldots, x_{n}\right) / M_{r}\left(x_{1}, x_{2}, \ldots, x_{n}\right) .\end{aligned}$

(a) Assume that $\mathrm{rs} \neq 0$. Let $X^{*}=\left(x_{1}^{*}, x_{2}^{*}, \ldots\right.$, $\left.x_{n}^{*}\right) \epsilon I$ be such that $F\left(X^{*}\right)=\max \{F(X): X \epsilon I\}$.

We shall first show that $X^{*}$ is a vertex of $I$. Indeed, suppose that this is not the case, and let $j$ be such that $A<x_{j}^{*}<B$. For every $x \in[A, B]$, let

$$
f(x)=F\left(x_{1}^{*}, x_{2}^{*}, \ldots, x_{j-1}^{*}, x, x_{j+1}^{*}, \ldots, x_{n}^{*}\right) .
$$

Then

$$
f\left(x_{j}^{*}\right)=\max \{f(x): A \leq x \leq B\},
$$

and therefore $f^{\prime}\left(x_{j}^{*}\right)=0$. Now a direct calculation shows that, throughout $(A, B), f^{\prime}(x)$ is of the form

$f^{\prime}(x)=\chi(x)\left[x^{s-r} \sum_{\substack{k=1 \\ k \neq j}}^{n} q_{k} x_{k}^{* r}-\sum_{\substack{k=1 \\ k \neq j}}^{n} q_{k} x_{k}^{* s}\right]$, where $\chi(x)>0$.

For $x=x_{j}^{*}$, the expression in the last square brackets vanishes; consequently, for every $x \epsilon\left(A, x_{j}^{*}\right)$ it is negative, and for every $x \epsilon\left(x_{j}^{*}, B\right)$ it is positive. Thus $f\left(x_{j}^{*}\right)<f(x)$ for every $x \in[A, B]$ distinct from $x_{j}^{*}$, contradicting (8).

For every $u \epsilon[0,1]$, let

$$
\left.\begin{array}{rl}
G(u) & =\left[u B^{s}+(1-u) A^{s}\right]^{1 / s}\left[u B^{r}+(1-u) A^{r}\right]^{-1 / r} \\
& =\left[u\left(\gamma^{s}-1\right)+1\right]^{1 / s}\left[u\left(\gamma^{r}-1\right)+1\right]^{-1 / \tau} .
\end{array}\right\}
$$


Since $X^{*}$ is a vertex of $I, F\left(X^{*}\right)=G\left(u^{*}\right)$ for some $u^{*} \epsilon[0,1]$. Thus, for every $X \epsilon I, F(X) \leq G\left(u^{*}\right)$; and, for every point $X$ of $I$ which is not a vertex, $F(X)<\dot{G}\left(u^{*}\right)$.

From (9) a straightforward computation shows that, throughout $(0,1), G^{\prime}(u)$ is of the form

$$
G^{\prime}(u)=\psi(u)(u-\Theta) \text {, where } \psi(u)<0 .
$$

As $G(0)=G(1)=1$, we must have, by Rolle's theorem, $0<\theta<1$. Since $G^{\prime}(u)>0$ throughout $(0, \theta)$ and $G^{\prime}(u)<0$ throughout $(\theta, 1)$, we have $G(u)<G(\theta)=\Delta$ for every $u \epsilon[0,1]$ distinct from $\theta$. From this follows the validity of (1) and of the necessary and sufficient condition at the end of the theorem.

(b) Assume that $\mathrm{r}=0<\mathrm{s}$. For every $\rho(\rho<s, \rho \neq 0)$ and for every $X \epsilon I$, we have, as proved above,

$M_{s}(X) / M_{\rho}(X) \leq$

$$
\left\{\frac{\rho\left(\gamma^{s}-\gamma^{\rho}\right)}{(s-\rho)\left(\gamma^{\rho}-1\right)}\right\}^{1 / s}\left\{\frac{s\left(\gamma^{\rho}-\gamma^{s}\right)}{(\rho-s)\left(\gamma^{s}-1\right)}\right\}^{-1 / \rho}
$$

By taking limits as $\rho \rightarrow 0$, we obtain, for every $X \in I$,

$$
M_{s}(X) / M_{0}(X) \leq\left\{\frac{\gamma^{s /\left(\gamma^{s}-1\right)}}{e \log \left\{\gamma^{s /\left(\gamma^{s}-1\right)}\right\}}\right\}^{1 / s},
$$

which proves (1). We shall, however, reprove it using the method of part (a), as that method yields the necessary and sufficient condition of the theorem for the present case.

Let $X^{* *}$ be a point of $I$ such that $F\left(X^{* *}\right)$ $=\max \{F(X): X \epsilon I\}$. As before, one shows that $X^{* *}$ is a vertex of $I$.

For every $u \epsilon[0,1]$, let

$$
\begin{aligned}
H(u)=\left\{u B^{s}+(1-u) A^{s}\right\}^{1 / s} /( & \left.B^{u} A^{1-u}\right) \\
& =\gamma^{-u}\left\{u\left(\gamma^{s}-1\right)+1\right\}^{1 / s} .
\end{aligned}
$$

Since $X^{* *}$ is a vertex of $I, F\left(X^{* *}\right)=H\left(u^{* *}\right)$ for some $u^{* *} \epsilon[0,1]$. Thus, for every $X \epsilon I, F(X) \leq H\left(u^{* *}\right)$; and, lor every point $X$ of $I$ which is not a vertex, $F(X)<H\left(u^{* *}\right)$. Again, considerations as in part (a) show that, for every $u \in[0,1]$ which differs from $\theta=(s \log \gamma)^{-1}-\left(\gamma^{s}-1\right)^{-1}(0<\theta<1)$, we have $H(u)$ $\langle H(\theta)=\Delta$. From here, again, follows (1) as well as the validity of the necessary and sufficient condition .

(c) Assume, finally, that $\mathrm{r}<0=\mathrm{s}$. This case can be handled by the method of part (a) and also by making direct use of the validity of the theorem in case (b).

The reader will notice the limit relations among the expressions for $\Delta$ given by (2) to (4) and among the expressions for $\Theta$ given by (5) to (7).

4. We close with a proof of the inequality between the arithmetic and the geometric means. Let $x_{1}, x_{2}, \ldots, x_{n}$ be arbitrary positive numbers, not all equal. Let $\alpha=\max \left\{-x_{k}: 1 \leq k \leq n\right\}$, and consider the function

$$
E(x) \equiv \prod_{k=1}^{n}\left(x+x_{k}\right)^{q_{k}} \quad(x>\alpha) .
$$

We observe that $M_{0}\left(x_{1}, x_{2}, \ldots, x_{n}\right) / M_{-1}\left(x_{1}, x_{2}\right.$, .. ., $\left.x_{n}\right)=E^{\prime}(0)$, and therefore a direct proof that $E^{\prime}(0)>1$ will yield (upon replacing each $x_{k}$ by $1 / x_{k}$ ) a proof of the desired inequality.

Now, for every $x>\alpha$, we have

$$
\frac{E^{\prime \prime}(x)}{E(x)}-\left(\frac{E^{\prime}(x)}{E(x)}\right)^{2}=\left(\frac{E^{\prime}(x)}{E(x)}\right)^{\prime}=-\sum_{k=1}^{n} q_{k} /\left(x+x_{k}\right)^{2} ;
$$

so that, by Cauchy's inequality,

$$
\frac{E^{\prime \prime}(x)}{E(x)}=\left(\sum_{k=1}^{n} q_{k}^{\frac{1}{2}} \frac{q_{k}^{\frac{1}{2}}}{x+x_{k}}\right)^{2}-\sum_{k=1}^{n} \frac{q_{k}}{\left(x+x_{k}\right)^{2}}<0 .
$$

Thus $E^{\prime}(x)$ is strictly monotone decreasing in $(\alpha, \infty)$; and, since $\lim _{x \rightarrow \infty} E^{\prime}(x)=1$, we have $E^{\prime}(0)>1$.

\section{References}

[1] Edwin F. Beckenbach and Richard Bellman, Inequalities, Springer-Verlag (1961).

[2] G. H. Hardy, J. E. Littlewood, and G. Pólya, Inequalities (second ed.), Cambridge Univ. Press (1952).

[3] Peter Henrici, Two remarks on the Kantorovich inequality, Am. Math. Monthly, 68, 904-906 (1961). 